\title{
Inducible gene manipulations in serotonergic neurons
}

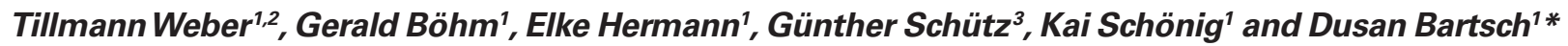 \\ 1 Department of Molecular Biology, Central Institute of Mental Health, Heidelberg University, Mannheim, Germany \\ 2 Department of Addictive Behavior and Addiction Medicine, Central Institute of Mental Health, Heidelberg University, Mannheim, Germany \\ ${ }^{3}$ Division Molecular Biology of the Cell I, German Cancer Research Center, Heidelberg, Germany
}

Edited by:

William Wisden, Imperial College, UK

\section{Reviewed by:}

Gregg E. Homanics,

University of Pittsburgh, USA;

Bernhard Lüscher, Pennsylvania State

University, USA

\section{*Correspondence:}

Dusan Bartsch, Department of Molecular Biology, Central Institute of Mental Health, Heidelberg University, J5, 68159 Mannheim, Germany. e-mail:dusan.bartsch@zi-mannheim.de
An impairment of the serotonergic (5-HT) system has been implicated in the etiology of many neuropsychiatric disorders. Despite the considerable genetic evidence, the exact molecular and pathophysiological mechanisms underlying this dysfunction remain largely unknown. To address the lack of instruments for the molecular dissection of gene function in serotonergic neurons we have developed a new mouse transgenic tool that allows inducible Cre-mediated recombination of genes selectively in $5-\mathrm{HT}$ neurons of all raphe nuclei. In this transgenic mouse line, the tamoxifen-inducible CreERT2 recombinase is expressed under the regulatory control of the mouse tryptophan hydroxylase 2 (Tph2) gene locus (177 kb). Tamoxifen treatment efficiently induced recombination selectively in serotonergic neurons with minimal background activity in vehicle-treated mice. These genetic manipulations can be initiated at any desired time during embryonic development, neonatal stage or adulthood. To illustrate the versatility of this new tool, we show that Brainbow-1.0L TPH2-CreERT2 mice display highly efficient recombination in serotonergic neurons with individual 5-HT neurons labeling with multiple distinct fluorescent colors. This labeling is well suited for visualization and tracing of serotonergic neurons and their network architecture. Finally, the applicability ofTPH2-CreERT2 for loxP-flanked candidate gene manipulation is evidenced by our successful knockout induction of the ubiquitously expressed glucocorticoid-receptor exclusively in 5-HT neurons of adult mice. The TPH2-CreERT2 line will allow detailed analysis of gene function in both developing and adult serotonergic neurons.

Keywords: serotonergic, tryptophan hydroxylase 2, serotonin, Cre-transgenic, knockout, mice, Brainbow, glucocorticoid-receptor

\section{INTRODUCTION}

Serotonergic neurons receive multiple, modulatory inputs from the hypothalamic-pituitary-adrenal (HPA) axis (Harfstrand et al., 1986; Day et al., 2004) and glutamatergic, GABAergic and other monoaminergic neurons (Sodhi and Sanders-Bush, 2004). Conversely, serotonergic (5-HT) neurons project to most structures of the brain (Hensler, 2006).

Serotonergic neurons modulate physiological functions including sleep, circadian rhythm, feeding, and neuroendocrine function (Hensler, 2006) as well as complex behaviors such as aggression (Miczek et al., 2007) or anxiety (Lucki, 1998). A multitude of evidence points to serotonergic dysregulation during development and adulthood in the etiology of many psychiatric diseases (Caspi et al., 2003; Mann, 2003; Gordon and Hen, 2004; Zill et al., 2004; Carver and Miller, 2006; Miczek et al., 2007; Mossner et al., 2007; Oades, 2007; Pardo and Eberhart, 2007; Carver et al., 2008; Geyer and Vollenweider, 2008; Serretti and Mandelli, 2008).

Furthermore, 5-HT plays a key role during development, being an essential neurotransmitter for proper neuronal division, differentiation, migration and synaptogenesis (Gaspar et al., 2003). Even transient alterations in serotonin homeostasis during development cause permanent changes to adult behavior (Ansorge et al., 2004; Gross and Hen, 2004).

In order to dissect the molecular basis of the multiplex serotonergic involvement in a plethora of physiological and pathophysiological brain functions, researchers have turned to the specificity offered by gene knockout technologies in mice. Unfortunately, germline knockouts can not address the specific serotonergic function of widely expressed genes without affecting the gene in other tissues as well. Moreover, germline gene inactivation may result in a lethal phenotype or induction of compensatory, homeostatic mechanisms or pleiotropy during development. Therefore, delineation of the particular function of the serotonergic system in relationship to other neuronal systems as well as the exact developmental and adult role of genes in 5-HT neurons remains elusive.

To overcome these obstacles, we have developed a CreERT2/ loxP-recombination system that allows temporal control of conditional gene manipulation specifically in serotonergic neurons. Temporal control of recombination is a prerequisite for distinguishing the developmental role of a gene from its present function during adulthood. For that reason, we took advantage of a fusion protein consisting of Cre recombinase and a mutated ligand-binding domain (LBD) of the human estrogen receptor (ER) that was developed to achieve tamoxifen dependent Cre activity (Feil et al., 1997; Indra et al., 1999).

We have chosen regulatory sequences of the tryptophan hydroxylase 2 (Tph2) gene which is exclusively expressed in serotonergic neurons during development and adulthood (Cote et al., 2003, 2007) to accurately direct Cre expression to 5-HT neurons of the brain. Transgenic TPH2-CreERT2 mice were generated by DNA microinjection of a modified DNA construct containing the Tph2 open reading frame together with large upstream and downstream 
flanking regions (177 kb). Breeding to three different mouse lines with loxP-flanked alleles shows reliable and efficient serotonergic neuron specific induction of recombination following tamoxifen treatment during development and adulthood and so represents a new and powerful tool for conditional gene manipulation in the serotonergic system.

\section{MATERIALS AND METHODS}

\section{GENERATION OF TPH2-CreERT2 TRANSGENIC MICE}

A 196-kb PAC (RP24-243J21, RZPD, Deutsches Ressourcenzentrum für Genomforschung $\mathrm{GmbH}$ ) that contains the full-length mouse Tph2 gene (107 kb) with $51 \mathrm{~kb}$ upstream and $19 \mathrm{~kb}$ downstream DNA sequences was selected for recombineering in EL250 bacteria (Lee et al., 2001). First, the kanamycin resistance gene of the pPAC4-backbone was replaced with a chloramphenicol resistance. Next, a cassette encoding a fusion protein (CreERT2) consisting of a Cre-recombinase (Cre) and a mutated LBD of the human ER (ERT2) as well as a kanamycin resistance gene flanked by two FRT sites was integrated into the ATG-start codon of the TPH2-gene. A 23-bp sequence downstream of the ATG-translation start of Tph2 was intentionally deleted since it contained additional in-frame ATG-start sites in Exon 1 (Figure S1 in Supplementary Material). The FRT-flanked kanamycin resistance cassette was then deleted by arabinose-induced expression of Flp recombinase. The CreERT2modified genomic Tph 2 sequence was separated from the PAC backbone by NotI digestion and subsequent preparative pulse-field electrophoresis. The purified, linearized DNA was microinjected into the pronucleus of C57BL/6N mouse oocytes. Transgenic offspring (founders) were identified by PCR genotyping of tail DNA. TPH2CreERT2 transgenic mice were always crossed with C57BL/6N mice periodically purchased from Charles River Laboratories.

The TPH2-CreERT2-transgenic mice were bred with R26R (Soriano, 1999), Brainbow1.0L (Livet et al., 2007) and GRflox (Tronche et al., 1999) mice to generate transgenic mice for recombination analysis: $\mathrm{R}^{26 \mathrm{R}^{\mathrm{TPH} 2-\mathrm{CreERT}}}{ }^{\text {, Brainbow1.0L }} \mathrm{L}^{\mathrm{TPH} 2-\mathrm{CrEERT}}$, and GRflox/GRflox ${ }^{\text {TPH2-CreERT2 }}$. To this purpose, TPH2-CreERT2 mice were crossed with $\mathrm{R} 26 \mathrm{R}$ and Brainbow mice to generate double transgenic R26R $\mathrm{R}^{\mathrm{TPH} 2-\mathrm{Cr} e \mathrm{RT} 2}$ and Brainbow1.0L $\mathrm{L}^{\mathrm{TPH} 2-\mathrm{CreERT} 2}$ mice, respectively. To generate GRflox/GRflox ${ }^{\text {TPH2-CreRT2 }}$ mice we first crossed TPH2-CreERT2 mice with homozygous GRflox/ GRflox mice. GRflox ${ }^{\text {TPH2-CreER2 }}$ mice were then crossed again with homozygous GRflox/GRflox mice to finally generate GRflox/ GRflox $^{\text {TPH2-CreERT2 mice. }}$

\section{IN VIVO INDUCTION OF Cre-MEDIATED RECOMBINATION WITH TAMOXIFEN}

Tamoxifen (Sigma) was dissolved in a corn oil/ethanol (10:1) mixture at a final concentration of $10 \mathrm{mg} / \mathrm{ml}$. Adult R26R $\mathrm{R}^{\text {TPH2-CreERT2 }}$ and GRflox/GRflox ${ }^{\text {TPH2-CreERT2 }}$ mice (8-12 weeks) were injected intraperitoneally twice daily for five consecutive days (protocol 1) with $1 \mathrm{mg}$ of tamoxifen. Adult Brainbow1.0L $\mathrm{L}^{\mathrm{TPH} 2-\mathrm{CreERT2}}$ mice $(8-12$ weeks) were injected intraperitoneally twice daily for one to five consecutive days. Control animals were injected with $100 \mu \mathrm{l}$ of a corn oil/ethanol vehicle mixture (vehicle). Experimental animals for immunohistochemistry (IHC) or X-gal staining were sacrificed 5-14 days after the last injection. For recombination analysis during embryogenesis, pregnant R26R ${ }^{\text {TPH2-CreERT2 }}$ mothers were injected for four consecutive days between E15 and E18 with $1 \mathrm{mg}$ tamoxifen twice daily. Offspring were delivered by $\mathrm{C}$-section at E20 and raised by foster mothers. For recombination analysis in postnatal pups, lactating transgenic mothers were injected intraperitoneally for five consecutive days with either $1 \mathrm{mg}$ tamoxifen twice daily (protocol 1), with $2 \mathrm{mg}$ tamoxifen twice daily (protocol 2), with $3 \mathrm{mg}$ tamoxifen twice daily (protocol 3) or one single injection of $1 \mathrm{mg}$ tamoxifen daily (protocol 4). $\mathrm{R} 26 \mathrm{R}^{\mathrm{TPH} 2-\mathrm{CreERT} 2}$ litters from embryonal and postnatal tamoxifen injections were analyzed at P60 for $\beta$-gal expression as described below. All experimental procedures were approved by the Animal Welfare Committee (Regierungspräsidium Karlsruhe) and carried out in accordance with the local Animal Welfare Act and the European Communities Council Directive of 24 November 1986 (86/609/EEC).

\section{X-GAL STAINING AND IMMUNOHISTOCHEMISTRY}

Transgenic mice were characterized by X-Gal staining and duallabel fluorescent IHC. The following primary antibodies were used: rabbit $\alpha$-TPH2 (kindly provided by Donald M. Kuhn, 1:10000), chicken $\alpha$ - $\beta$-galactosidase (Abcam, 1:10000), rabbit $\alpha$-GR (Santa Cruz Biotechnology, 1:1000) and mouse $\alpha$-TPH1 (Sigma, 1:500-2000) antibodies. The TPH1 antibody cross-reacts with TPH2 and detects both isoenzymes. Secondary antibodies were AF488 donkey $\alpha$-rabbit (Invitrogen, 1:1000) and AF488 donkey $\alpha$-mouse (Invitrogen, 1:200), AF555 donkey $\alpha$-rabbit (Invitrogen, 1:1000), and Cy3 donkey $\alpha$-chicken (Jackson ImmunoResearch, 1:1000). Sections were examined using a Nikon C1Si-CLEM confocal laser-scanning microscope (Nikon Imaging Center, BioQuant, Heidelberg, Germany). Confocal image stacks for both channels were acquired sequentially, and projected on average using ImageJ software. For the combinatorial fluorescent expression in Brainbow1.0L ${ }^{\text {TPH2-CreERT2 }}$ mice a Leica TCS SP5 X confocal laser-scanning microscope (Leica, Mannheim, Germany) was employed. For cyan fluorescent protein (CFP) excitation a 458-nm argon line was used. Excitation of yellow fluorescent protein (YFP) and RFP was performed with a white light laser at 512 and $565 \mathrm{~nm}$, respectively. Confocal image stacks for all channels were acquired sequentially, and maximally projected using LAS AF Lite software. Confocal images were not processed with Adobe Photoshop to intensify colors and accentuate hues.

\section{STATISTICAL METHODS}

Coronal slices of two to four adult R26R ${ }^{\text {TPH2-CreERT2 }}$ mice of each founder line were processed with dual-label fluorescent IHC detecting $\beta$ gal and TPH2. Image stacks of all slices that showed TPH2 staining were acquired using a confocal laser-scanning microscope. The ratio of $\beta$ gal+/TPH $2+$ neurons to all $\mathrm{TPH} 2+$ neurons was calculated separately for caudal, median and dorsal raphe nuclei. Confidence-bounds (CI) for recombination efficacy and background recombination in adult mice were calculated using the Clopper-Pearson method based on significance level 95.0\% (Clopper and Pearson, 1934). For adult R26R ${ }^{\text {TPH2-CreERT2 }}$ mice which had been injected during E15-18, P2-6 and P12-16 $(n=3$ each group) the ratio of $\beta$ gal $+/ \mathrm{TPH} 2+$ neurons to all TPH $2+$ neurons per group was expressed as the mean and as the range of expression. 


\section{RESULTS GENERATION OF TPH2-CreERT2 TRANSGENIC MICE}

We used the CreERT2/loxP-system which provides inducible, Cre-mediated recombination upon tamoxifen administration to generate transgenic mice which allow conditional manipulation of individual genes in serotonergic neurons at any desired time. Targeted spatial control in serotonergic neurons was achieved by using elements of the Tph2 locus as a promoter to control Cre expression. Since precise information about the regulatory elements which govern timely and 5-HT specific TPH2 expression is not available, we used a large genomic fragment of the Tph2 locus to drive CreERT2, thereby enhancing the probability to include most of the important regulatory components of Tph2. To this end, a P1-derived artificial chromosome (pPAC4) with a $177 \mathrm{~kb}$ genomic insert containing the entire Tph 2 gene with large additional flanking sequences (see Section "Materials and Methods") was used to generate the TPH2-CreERT2 construct. The coding sequence of the CreERT2 fusion protein was inserted in place of the ATG-start of the Tph 2 gene (Figure 1) by recombineering in bacteria (Lee et al., 2001). The modified genomic fragment was released from the PAC backbone and finally introduced into the mouse germline of C57BL/6N by DNA microinjection. Five TPH2-CreERT2 founders were obtained $(1.21,1.23,1.27,1.35$ and 1.38).

\section{EFFICIENT AND SPECIFIC RECOMBINATION IN SEROTONERGIC NEURONS OF ADULT R26R ${ }^{\text {Tph2CreERT2 }}$ MICE}

TPH2-CreERT2 founder were crossed to ROSA26 Cre reporter mice (R26R) (Soriano, 1999) to obtain R26R ${ }^{\text {Tph2CreERT2 mice. Temporal }}$ and spatial control of tamoxifen-induced CreERT2-mediated recombination in 5-HT neurons was determined and the efficacy of serotonergic recombination in the caudal, dorsal and median raphe (MR) nuclei was calculated.

Adult R26 $\mathrm{R}^{\mathrm{Tph} 2 \mathrm{CreERT} 2}$ mice were injected twice daily with either tamoxifen ( $1 \mathrm{mg}$ ) or vehicle for five consecutive days. Animals were sacrificed for analysis 5 days after the last tamoxifen injection. X-Gal staining of tamoxifen-treated R26R ${ }^{\mathrm{Tph} 2 \mathrm{CreERT} 2}$ mice from all lines revealed recombination in the brain stem and midbrain in regions shown to contain serotonergic somata (Figures $2 \mathrm{~A}-\mathbf{I}$ ) while extraserotonergic brain regions showed no staining. Vehicle treated $\mathrm{R} 26 \mathrm{R}^{\mathrm{Tph} 2 \mathrm{CreERT2}}$ mice showed minimal background recombination

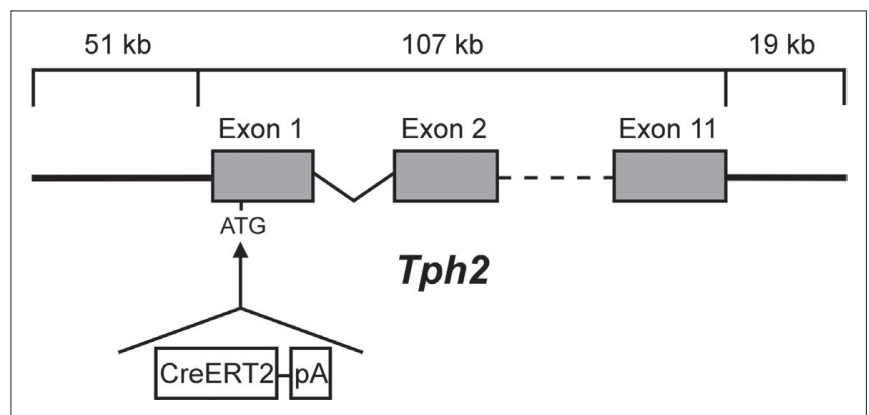

FIGURE 1 |TPH2-CreERT2 construct for DNA microinjection.

A PCR-amplified CreERT2.pA cassette flanked by sequences (42 bp) homologous to the integration site of the PAC-based genomic Tph2 locus was recombineered in E. coli. CreERT2.pA was inserted into the ATG-start of Exon 1 of the mouse Tph2 gene. among all lines (Figures 2J-L). TPH2-CreERT2 lines 1.21, 1.35 and 1.38 showed the highest recombination efficiency and lowest background recombination.

Next, we performed dual-label fluorescent IHC with anti- $\beta$-galactosidase $(\beta$ gal $)$ and anti-TPH2-antibodies to investigate selectivity and efficiency of recombination in serotonergic neurons. While $\beta$-gal antibodies confirm Cre-mediated recombination in the Rosa26 locus, TPH2 antibodies detect TPH2 which is the rate-limiting enzyme in brain serotonin synthesis and solely found in serotonergic raphe nuclei. As suggested by X-gal staining, recombination occurred exclusively in serotonergic neurons of all raphe nuclei with comparable efficiency (Figures 3A-I). Vehicle treated mice showed only minimal background recombination (Figures $3 \mathrm{~J}-\mathrm{L}$ ).

Lines 1.21 and 1.38 showed the highest recombination efficacy in serotonergic neurons when tamoxifen was injected (91 and 90\%, respectively) (Table S1 in Supplementary Material) while background recombination in vehicle treated mice was below 2\% (Table S2 in Supplementary Material). All further experiments were carried out with line 1.38 .

\section{RECOMBINATION IN SEROTONERGIC NEURONS OF R26R ${ }^{\text {TPH2CreERT2 }}$ MICE DURING EMBRYOGENESIS AND POSTNATAL DEVELOPMENT}

To investigate whether tamoxifen inducible recombination is also feasible during embryogenesis we injected pregnant mothers intraperitoneally with $1 \mathrm{mg}$ tamoxifen twice daily for four consecutive days between E15 and E18. Offspring were delivered by C-section

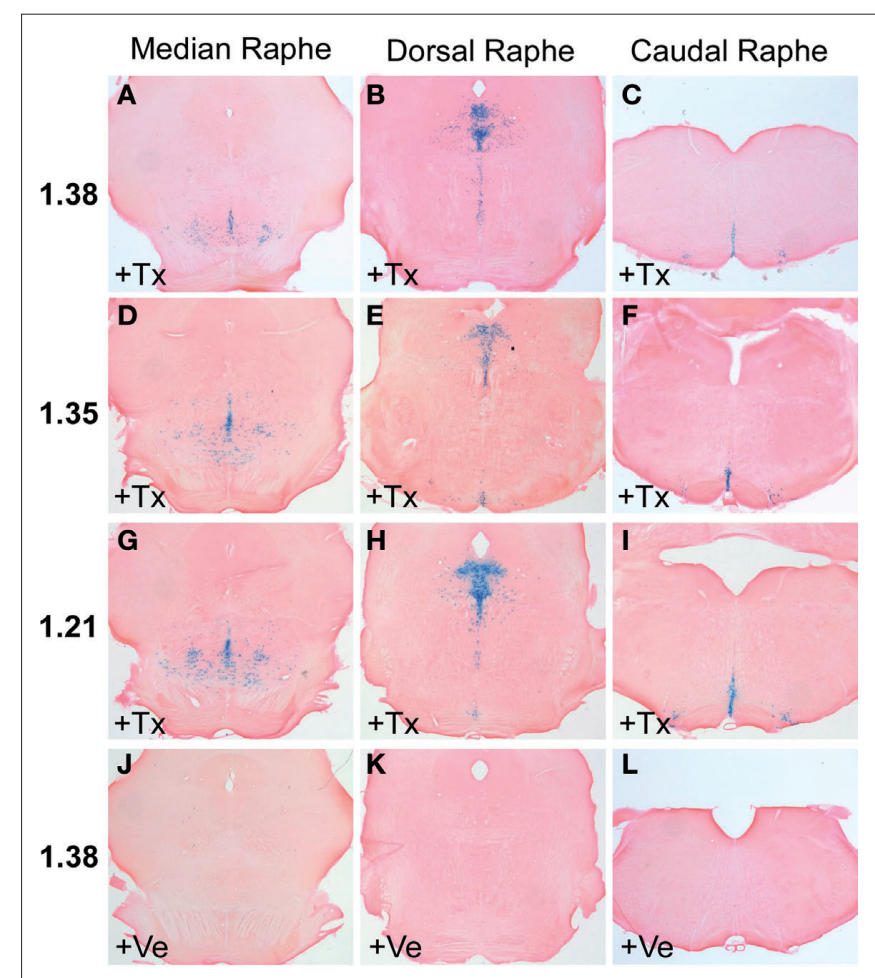

FIGURE 2 |X-Gal staining of R26 ${ }^{\text {TPH2-CreERT2 }}$ mice is restricted to the brain stem and midbrain where raphe nuclei are located. $(\mathbf{A}-\mathbf{C}, \mathbf{J}-\mathbf{L})$ Line 1.38 . (D-F) Line 1.35. (G-I) Line 1.21. (A-I) Mice treated with tamoxifen (+Tx) (1 mg twice daily for 5 days) show abundant $X-G a l$ staining at all levels of raphe nuclei. (J-L) Vehicle (+Ve) treated mice show nonsignificant $X-G a l$ staining. (A,D,G,J) Median raphe nuclei; $(\mathbf{B}, \mathbf{E}, \mathbf{H}, \mathbf{K})$ dorsal raphe nuclei; $\mathbf{( C , F , I , L )}$ caudal raphe nuclei. 


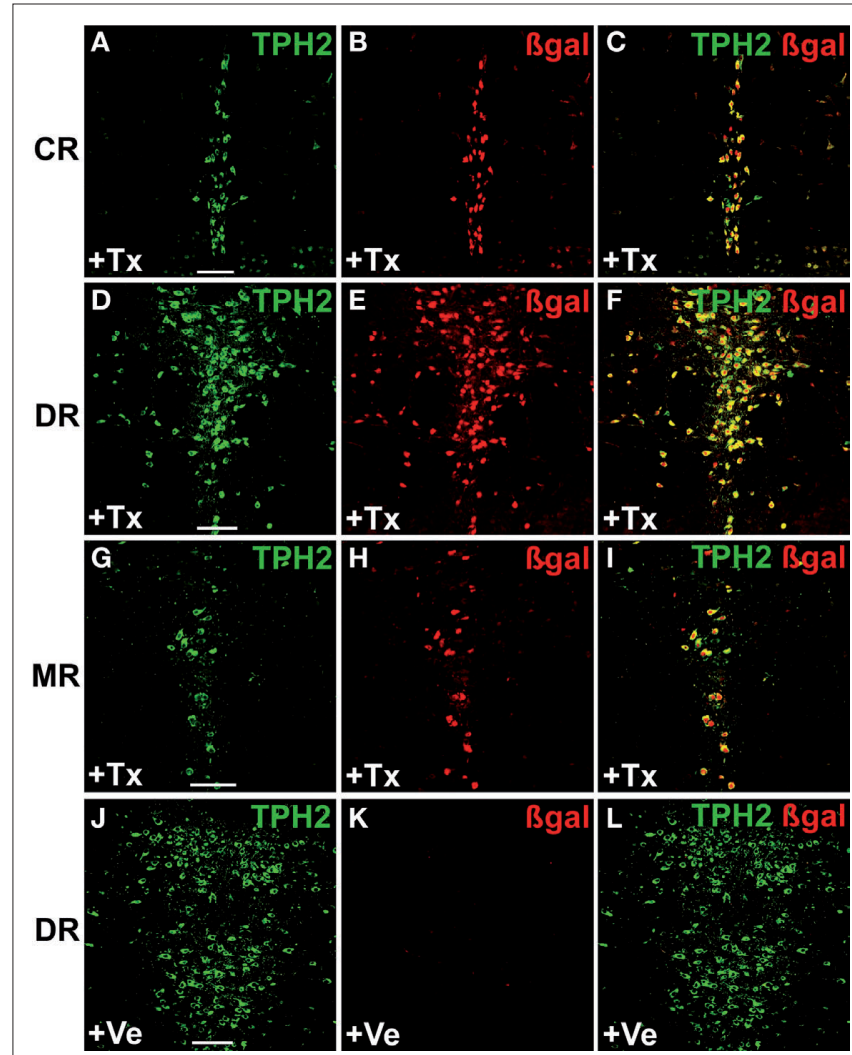

FIGURE 3 | Inducible recombination is restricted to serotonergic neurons of adult R26R $\mathbf{R}^{\text {TPH2-CreERT2 }}$ mice. R26R ${ }^{\text {TPH2-CreERT2 }}$ mice of line 1.38 were injected with tamoxifen (1 mg twice daily) or vehicle for five consecutive days starting at P90. Coronal sections show dual-label fluorescence immunohistochemistry for TPH2 and $\beta$-gal in tamoxifen-treated $(+\mathrm{TX})(\mathbf{A}-\mathbf{I})$ and vehicle-treated mice (+Ve) (J-L). (A,D,G,J) TPH2 fluorescence; $(\mathbf{B}, \mathbf{E}, \mathbf{H}, \mathbf{K}) \mathbf{\beta}$-gal fluorescence; $(\mathbf{C}, \mathbf{F}, \mathbf{I}, \mathbf{L})$ Merged images. Colocalization is visualized at the level of caudal raphe (CR) nuclei (A-C), dorsal raphe (DR) nuclei (D-F,J-L) and median raphe nuclei (MR) (G-I) using confocal images. (C,F,I) In tamoxifen-treated mice there is extensive colocalization of $\beta$-gal and $\mathrm{TPH} 2$ indicating efficient recombination (J-L) Vehicle-treated mice show minimal colocalization demonstrating low background recombination without tamoxifen. Scale bars: $100 \mu \mathrm{m}$.

on E20 since tamoxifen treatment impairs parturition (Boyle et al., 2008; Erdmann et al., 2008). R26R $\mathrm{R}^{\mathrm{TPH} 2-\mathrm{CreERT} 2}$ offspring were sacrificed in adulthood and the efficacy and selectivity of recombination analyzed with IHC against TPH 2 and $\beta$-gal. Serotonergic recombination could be efficiently induced with tamoxifen during embryogenesis (recombination efficacy 66\%) (Figures 4A-C; Figures S2A-C,J-L in Supplementary Material; Table S3 in Supplementary Material) but recombination rates were below those achieved during adulthood.

Next, we determined whether recombination during early and later postnatal development is feasible. Lactating mothers were injected intraperitoneally for five consecutive days starting at P2 and at P12, respectively. Different tamoxifen protocols were used: $1 \mathrm{mg}$ tamoxifen twice daily (protocol 1), 2 mg tamoxifen twice daily (protocol 2), $3 \mathrm{mg}$ tamoxifen twice daily (protocol 3) and $1 \mathrm{mg}$ tamoxifen once daily (protocol 4).

Intraperitoneal injections were well tolerated by lactating mothers during the five consecutive days of tamoxifen treatment but mothers tended to abandon their offspring thereafter,

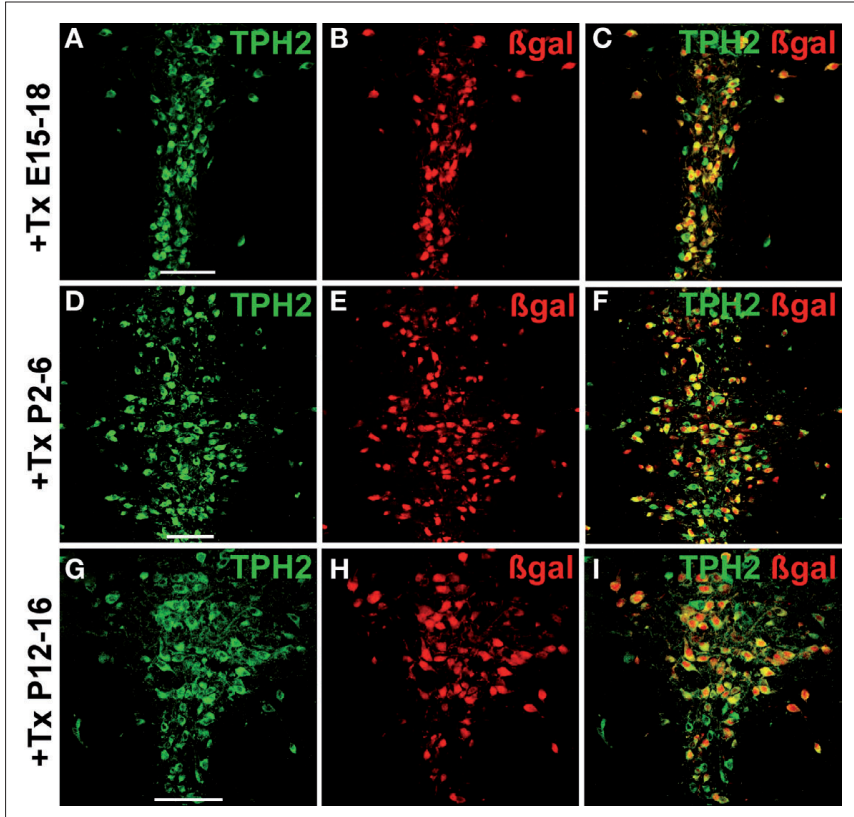

FIGURE 4 | Inducible recombination in serotonergic neurons of embryonal and postnatal $\mathbf{R 2 6 R}^{\text {TPH2-CreERT2 }}$ mice. Pregnant or lactating mothers of line 1.38 were injected with tamoxifen $(+T x)$. (A-C) One milligram twice daily at E15-18; (D-F) 1 mg twice daily at P2-6; (G-I) 3 mg twice daily at $\mathrm{P} 12-16$. Transgenic R26R $\mathrm{R}^{\text {TPH2-CreERT2 }}$ offspring were sacrificed at P60 and analyzed with dual-label fluorescence immunohistochemistry for TPH2 and $\beta$-gal. Colocalization is visualized at the level of dorsal raphe nuclei using confocal images. (A,D,G) TPH2 fluorescence; (B,E,H) $\beta$-gal fluorescence; $(\mathbf{C}, \mathbf{F}, \mathbf{I})$ merged images. Frequent colocalization of $\beta$-gal and TPH 2 confirms efficient recombination during embryogenesis (C) and early (F) and later (I) postnatal development. Scale bars: $100 \mu \mathrm{m}$.

in particular those treated with $2 \mathrm{mg} \times 2 \mathrm{mg}$ tamoxifen during early postnatal development (P2-6). Recombination during early and later postnatal development could be successfully induced in serotonergic neurons (Figures 4D-I; Figures S2D-I,M-R in Supplementary Material) but recombination efficiency varied significantly with protocols. Tamoxifen injections twice daily $(2 \mathrm{mg} \times 1 \mathrm{mg})$ led to superior recombination efficacy $(68 \%)$ compared to single daily injections $(1 \mathrm{mg} \times 1 \mathrm{mg})$ whereas doubling the amount of tamoxifen $(2 \mathrm{mg} \times 2 \mathrm{mg})$ during early postnatal development (P2-6) did not result in better recombination efficacy than with $2 \mathrm{mg} \times 1 \mathrm{mg}$ tamoxifen per day. In contrast, further increasing the amount of tamoxifen $(2 \mathrm{mg} \times 3 \mathrm{mg})$ in lactating mothers with older offspring (P12-16) led to recombination efficacies (72\%) comparable to those achieved during early postnatal development with $2 \mathrm{mg} \times 1 \mathrm{mg}$ tamoxifen (Table S3 in Supplementary Material).

In conclusion, significant recombination efficacies could be attained in R26R ${ }^{\text {TPH2-CreERT2 }}$ offspring from tamoxifen injected pregnant or lactating mothers but recombination rates did not reach the level of adult recombination (90\%). We also noted that insubstantial recombination occurred during embryogenesis and early postnatal development in regions which do not contain 5-HT neurons in adult mice. A few $\beta$-gal-positive/TPH2-negative cells were detected in the caudal and MR nuclei, the hypothalamus and deep layers of the cortex. 


\section{INDUCIBLE RECOMBINATION IN BRAINBOW-1.0L ${ }^{\text {TPH2-CreERT2 }}$ MICE RESULTS IN COMBINATORIAL EXPRESSION OF FLUORESCENT PROTEINS WITH MULTIPLE, DISTINCT COLORS IN 5-HT NEURONS}

The recently described Brainbow-1.0L transgenic mouse line (Livet et al., 2007) allows individual fluorescence labeling of living neurons. In this line, Cre-mediated recombination of loxP-flanked fluorescence transgenes leads to a switch from RFP (red fluorescent protein) expression to a stochastically determined combinatorial expression of CFP and YFP.

Double transgenic Brainbow-1.0L $\mathrm{L}^{\mathrm{TPH} 2-\mathrm{CreERT} 2}$ mice were induced with $1 \mathrm{mg}$ tamoxifen twice daily for one to five consecutive days and recombination was analyzed by fluorescence emission. Confocal laser-scanning microscopy of RFP, CFP and YFP fluorescence detected the combinatorial expression of CFP and YFP leading to mosaic fluorescent labeling of serotonergic neurons (Figure 5; Figure S3 in Supplementary Material). The length of tamoxifen treatment correlated with the proportion of recombined 5-HT neurons. Three days of tamoxifen treatment were sufficient to achieve maximal serotonergic recombination whereas in animals given 1-2 days of tamoxifen treatment the proportion of recombined 5-HT neurons was significantly reduced.

\section{EFFICIENT KNOCKOUT OF THE GLUCOCORTICOID-RECEPTOR GENE IN SEROTONERGIC NEURONS OF ADULT GRflox/GRflox ${ }^{\text {TPH2-CreERT2 }}$ MICE}

Both R26R and Brainbow mice are reporter mice which express fluorescent proteins or $\beta$ gal following Cre-mediated recombination. To demonstrate that loxP flanked target genes can also be efficiently deleted in the adult serotonergic system we generated TPH2-CreERT2 mice that are homozygous for the loxP-flanked

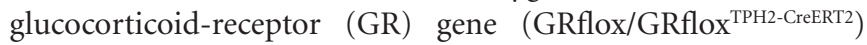
(Tronche et al., 1999). The GR protein is ubiquitously expressed throughout the brain. In particular, 5-HT neurons show abundant GR-antibody staining. Adult mice ( 2 months old) were treated with tamoxifen ( $1 \mathrm{mg}$ twice daily for 5 days) and sacrificed 14 days later. Double-immunostaining against GR and TPH revealed that
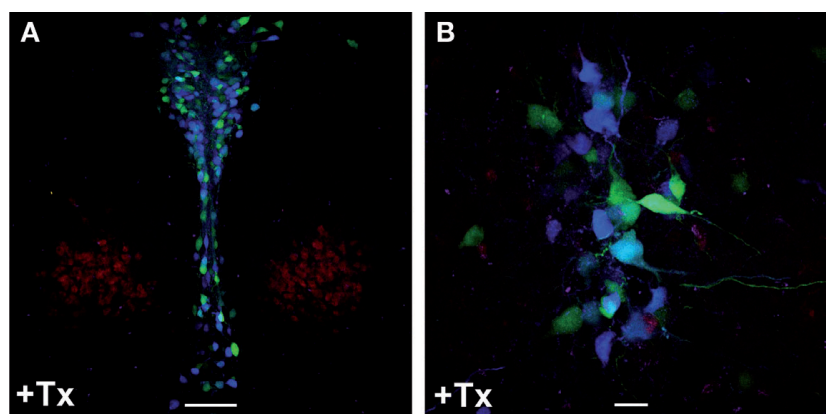

FIGURE 5 | Inducible recombination in adult Brainbow1.0L ${ }^{\text {TPH2-CreERT2 }}$ mice leads to combinatorial expression of CFP and YFP in serotonergic neurons. Tamoxifen (+Tx) (1 mg twice daily) was injected for five consecutive days starting at P90. (A,B) One week later, mice were sacrificed and red, cyan and yellow fluorescence emission was analyzed by confocal microscopy at the level of dorsal raphe nuclei. Stochastic recombination of loxP-flanked fluorescent genes leads to CFP and YFP expression. Depending on the ratio of CFP to YFP expression, each 5-HT neuron shows a distinct color ranging from dark blue (only CFP expression) to yellow (only YFP expression). (B) Fluorescence labeled neuronal projections are easily detectable. Scale bars: (A) $100 \mu \mathrm{m}$, (B) $20 \mu \mathrm{m}$.
80-90\% of 5-HT neurons had lost their GR-immunoreactivity whereas GR expression was unchanged in all other brain regions examined (Figure 6; Figure S4 in Supplementary Material).

\section{DISCUSSION}

In this study, we describe a novel transgenic tool for conditional somatic manipulation of loxP-flanked target genes in serotonergic neurons. Using the Tph2 genomic locus as a regulatory element to direct CreERT2 expression specifically to serotonergic neurons we show efficient, tamoxifen-inducible recombination with minimal background activity in R26R ${ }^{\mathrm{TPH} 2-\mathrm{CreERT} 2}$ mice during development and adulthood.

Traditionally, transgenic mouse lines are generated via pronuclear injection of plasmid-derived DNA resulting in random integration of the transgene into the genome. The level and pattern of plasmid-derived transgene expression is determined both by the promotor and its integration site in the genome. Such transgenic mice show copy-number independent transgene expression in regions or cell types not associated with the original specificity of the promoter (Bronson et al., 1996; Wallace et al., 2000; Chandler et al., 2007).

Because of the large insert size of BAC/PAC clones, they can often accommodate an entire gene of interest, including long-range cis-acting regulatory elements required for correct temporal and tissue-specific expression (Chandler et al., 2007). This is especially desirable for experiments where precise transgene expression is critical, such as for promotors driving Cre-recombinase (Lee et al., 2001). These large constructs allow copy-number dependent expression of the transgene resembling the expression of the endogenous gene independent of the integration site (Schedl et al., 1993; Casanova et al., 2001; Giraldo and Montoliu, 2001; Gong et al., 2003).
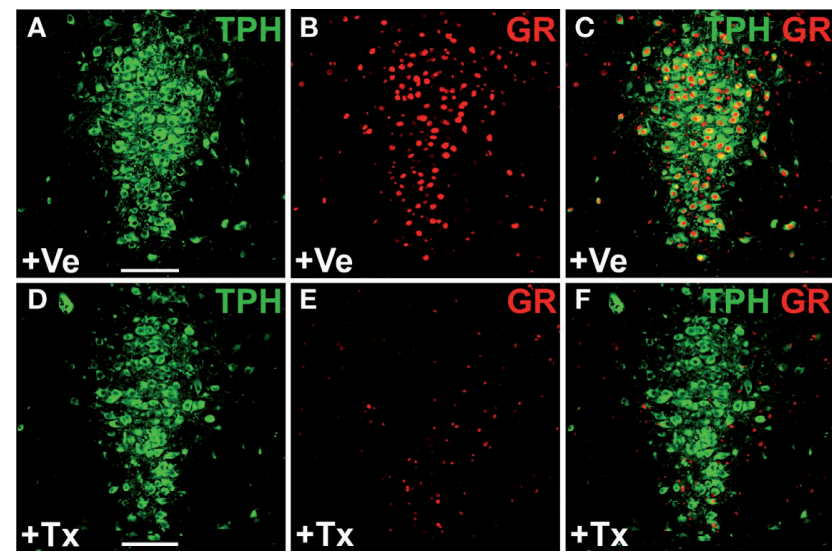

FIGURE 6 | Inducible glucocorticoid receptor (GR) knockout in serotonergic neurons of adult GRflox/GRflox ${ }^{\text {TPH2-CreERT2 }}$ mice. Tamoxifen (1 mg twice daily) or vehicle was injected for five consecutive days starting at P60. Coronal sections show dual-label fluorescence immunohistochemistry forTPH and GR in vehicle-treated (+Ve) (A-C) and tamoxifen-treated (+Tx)

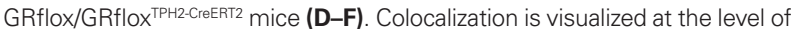
dorsal raphe nuclei using confocal images. (A,D)TPH fluorescence; (B,E) GR fluorescence; (C,F) merged images. (C) In vehicle-treated mice, GR colocalizes extensively with TPH. (F) GR is primarily expressed in TPH-negative neurons in tamoxifen-treated GR-knockout mice verifying the selective deletion of GR in 5-HT neurons. Scale bars: $100 \mu \mathrm{m}$. 
In this study we were able to demonstrate that tissue-specific regulatory elements of the mouse Tph 2 locus, present on a $177 \mathrm{~kb}$ genomic DNA fragment and comprising the entire Tph2 gene plus large flanking regions, reliably direct Cre expression selectively to serotonergic neurons in all five analyzed transgenic TPH2-CreERT2 lines.

To date only two mouse lines published have been shown to target loxP-flanked gene knockouts to the serotonergic system. A knockin of the Cre recombinase into the Sert gene has been used to achieve recombination in serotonergic neurons (Zhuang et al., 2005). However, this strategy is compromised by the fact that it results in a hemizygous Sert knockout. Furthermore, SERT is expressed in extraserotonergic neurons during development (Gaspar et al., 2003). Therefore, using the endogenous Sert locus to control Cre expression will lead to developmental recombination in neurons outside the serotonergic system. Finally, the Sert-Cre mouse line does not contain regulatory elements that allow temporal control of Cre-mediated recombination.

Recently, a Pet1-Cre transgenic mouse line was published (Scott et al., 2005) in which cell-type specific serotonergic gene inactivation of loxP-flanked target genes will be irrevocably initiated at E12.5 during embryogenesis. Despite its excellent characteristics to create tissue-specific embryonal knockouts in the serotonergic system, Pet1-Cre lacks the possibility of temporal control. As Pet-1 is a key player in a transcriptional mechanism that controls embryonal 5-HT neuron differentiation (Hendricks et al., 2003) it would be of interest whether its regulatory elements are sufficient to achieve inducible CreERT2-mediated gene inactivation in the entire serotonergic system of the postnatal and adult brain as well.

$\mathrm{TPH} 2$ is the rate-limiting enzyme of 5-HT synthesis in the serotonergic raphe nuclei (Walther et al., 2003). In the brain, it is strongly and exclusively expressed in serotonergic neurons starting during embryogenesis around E11 and continuing throughout adult life (Cote et al., 2003, 2007). Thus, regulatory elements of the Tph2 locus should ensure strong serotonergic Cre expression in TPH2-CreERT2 mice at any time from embryogenesis to adulthood. To test this, we induced recombination in $\mathrm{R} 26 \mathrm{R}^{\mathrm{TPH} 2-\mathrm{CreERT} 2}$ mice during embryogenesis, postnatal development and adulthood. In adult mice, tamoxifen injections twice daily for five consecutive days lead to highly efficient induction of recombination specifically in serotonergic neurons. During embryogenesis and postnatal development intraperitoneal injections to the mothers led to efficient serotonergic recombination $(66-72 \%)$ in their offspring but rates were inferior to adult recombination (90\%). This finding is most likely a result of the tamoxifen application route rather than of attenuated activity of Tph2 regulatory sequences driving CreERT2 during development as expression of TPH2 commences already at E11. Moreover, we used the identical Tph2 regulatory sequences to direct the expression of other genes to serotonergic neurons during development and found strong expression already at E15 (unpublished observation). In our opinion, these results demonstrate that ip application to the mother does not lead to sufficient tamoxifen concentrations in their offspring to achieve high recombination rates. Furthermore, tamoxifen is an active substance which inhibits ERs and stimulates progesterone receptors. In pregnant mice, tamoxifen treatment inhibits parturition (Boyle et al., 2008; Erdmann et al., 2008) which necessitated scheduled cesarean sections at E20. While behavioral effects of tamoxifen treatment have not been identified in adult mice (Vogt et al., 2008) there is no comparable data on mice available that received tamoxifen treatment during development.

Taken together, Pet1-Cre mice are preferable if embryonal serotonergic gene deletion starting at E12.5 (Scott et al., 2005) is desired as they do not require tamoxifen and convey high recombination rates. Inducible TPH2-CreERT2 mice are advantageous if recombination is to start later than E12.5. As such, TPH2-CreERT2 mice are currently the only alternative for postnatal and adult induction of serotonergic recombination. Nonetheless, further research needs to be conducted to improve tamoxifen application during development and to evaluate the biochemical, molecular and behavioral impact this treatment exerts on developing offspring.

Serotonergic recombination in Brainbow-1.0 $\mathrm{L}^{\mathrm{TPH} 2-\mathrm{CreERT} 2}$ mice (Livet et al., 2007) creates a stochastic choice of Thy1-promotor controlled expression between two different loxP-flanked fluorescence genes as a result of tandem integration of Brainbow$1.0 \mathrm{~L}$ copies. To date, it was unclear whether the Thy1-promotor is active in 5-HT neurons. Here we show that, upon tamoxifen induction, Brainbow-1.0L $\mathrm{L}^{\text {TPH2-CreERT2 }}$ mice display highly efficient recombination in 5-HT neurons. Virtually all serotonergic neurons can be labeled with combinatorial CFP and YFP expression. An advantage of the TPH2-CreERT2 system in conjunction with the Brainbow mice is the possibility of titrating the proportion of 5-HT neurons exhibiting recombination by reducing the duration of tamoxifen treatment. This should facilitate anterograde tracing experiments of single, distinctly color-labeled serotonergic cell projections by three-dimensional reconstruction from serial sections which will help to define the connectivity of individual 5-HT neurons or its cell clusters (B1-9) with other brain regions and within the raphe nuclei.

Tissue-specific recombination efficacies derived from artificial Cre reporter lines should be interpreted carefully regarding their value to predict recombination frequencies for loxP-flanked target genes (Weber et al., 2001). On this account, we substantiated our results obtained with R26R Cre reporter and Brainbow mice by inducing a selective knockout of the endogenous GR gene exclusively in 5-HT neurons of the adult mouse. With the GRflox/GRflox ${ }^{\text {TPH2-CreERT2 }}$ mice it will be possible to decipher the reciprocal interactions between the HPA axis and 5-HT neurons, systems that have been implicated together in the etiology of several pathological conditions like escalated aggression and violence or anxiety and depression (Haller et al., 2005; Miczek et al., 2007; Lanfumey et al., 2008).

The neurotransmitter serotonin plays a diverse and important role in both the developing and adult brain (Gaspar et al., 2003). With our TPH2-CreERT2 line we provide a tool for highly efficient and inducible recombination specifically in the serotonergic system. This system will allow the study of the roles of many genes in the regulation of serotonin systems and hence its role in brain development and function. Moreover, the possibility of manipulating gene expression selectively in mature serotonergic neurons allows direct molecular and behavioral effects of candidate gene deletion to be distinguished from compensatory, homeostatic mechanisms during development. In the pathology of complex neuropsychiatric diseases, serotonergic genes, in conjunction with stressors, may act at 
any time from development to adult life to predispose an individual to psychiatric disorders (Caspi et al., 2002, 2003; Champoux et al., 2002). The action of perturbed serotonergic genes may even produce opposite phenotypes depending on time of action (Gross and Hen, 2004). This TPH2-CreERT2 mouse model may help to delineate time windows in which gene inactivation is crucial for the pathogenesis of neuropsychiatric disorders dependent on serotonergic abnormalities. Furthermore, varying the onset of gene manipulation during different periods of life and combining genetic manipulation with pharmacological or behavioral interventions will help to unravel gene-environment interactions that are crucial for the development or maintenance of pathological phenotypes.

\section{REFERENCES}

Ansorge, M. S., Zhou, M., Lira, A., Hen, R., and Gingrich, J. A. (2004). Early-life blockade of the 5-HT transporter alters emotional behavior in adult mice. Science 306, 879-881.

Boyle, S., Misfeldt, A., Chandler, K. J., Deal, K. K., Southard-Smith, E. M., Mortlock, D. P., Baldwin, H. S., and de Caestecker, M. (2008). Fate mapping using Cited1-CreERT2 mice demonstrates that the cap mesenchyme contains self-renewing progenitor cells and gives rise exclusively to nephronic epithelia. Dev. Biol.313, 234-245.

Bronson, S. K., Plaehn, E. G., Kluckman, K. D., Hagaman, J. R., Maeda, N., and Smithies, O. (1996). Single-copy transgenic mice with chosen-site integration. Proc. Natl. Acad. Sci. U.S.A. 93, 9067-9072.

Carver, C. S., and Miller, C. J. (2006). Relations of serotonin function to personality: current views and a key methodological issue. Psychiatry Res. 144, 1-15.

Carver, C. S., Johnson, S. L., and Joormann, J. (2008). Serotonergic function, two-mode models of selfregulation, and vulnerability to depression: what depression has in common with impulsive aggression. Psychol. Bull. 134, 912-943.

Casanova, E., Fehsenfeld, S., Mantamadiotis, T., Lemberger, T., Greiner, E., Stewart, A. F., and Schutz, G. (2001). A CamKIIalpha iCre BAC allows brain-specific gene inactivation. Genesis 31, 37-42.

Caspi, A., McClay, J., Moffitt, T. E., Mill, J., Martin, J., Craig, I. W., Taylor, A., and Poulton, R. (2002). Role of genotype in the cycle of violence in maltreated children. Science 297, 851-854.

Caspi, A., Sugden, K., Moffitt, T. E., Taylor, A., Craig, I. W., Harrington, H., McClay, J., Mill, J., Martin, J., Braithwaite,A., and Poulton, R. (2003). Influence of life stress on depression: moderation by a polymorphism in the 5-HTT gene. Science 301, 386-389.
Champoux, M., Bennett, A., Shannon, C., Higley, J. D., Lesch, K. P., and Suomi, S. J. (2002). Serotonin transporter gene polymorphism, differential early rearing, and behavior in rhesus monkey neonates. Mol. Psychiatry 7, 1058-1063. Broeckelmann, E. M., Hou, Y., Mortlock, D. P. (2007). Relevance of BAC transgene copy number in mice: transgene copy number variation across multiple transgenic lines and correlations with transgene integrity and expression. Mamm. Genome 18, 693-708.

Clopper, C., and Pearson, S. (1934). The use of confidence or fiducial limits illustrated in the case of the binomial. Biometrika 26, 404-413.

Cote, F., Fligny, C., Bayard, E., Launay, J. M., Gershon, M. D., Mallet, J., and Vodjdani, G. (2007). Maternal serotonin is crucial for murine embryonic development. Proc. Natl. Acad. Sci. U.S.A. 104, 329-334.

Cote, F., Thevenot, E., Fligny, C., Fromes, Y., Darmon, M., Ripoche, M.A., Bayard, E., Hanoun, N., Saurini, F., Lechat, P., Dandolo, L., Hamon, M., Mallet, J., and Vodjdani, G. (2003). Disruption of the nonneuronal tphl gene demonstrates the importance of peripheral serotonin in cardiac function. Proc. Natl. Acad. Sci. U.S.A. 100, 13525-13530.

Day, H. E., Greenwood, B. N., Hammack, S. E., Watkins, L. R., Fleshner,M.,Maier,S.F., and Campeau, S. (2004). Differential expression of 5HT-1A, alpha $1 \mathrm{~b}$ adrenergic, CRF$\mathrm{R} 1$, and CRF-R2 receptor mRNA in serotonergic, gamma-aminobutyric acidergic, and catecholaminergic cells of the rat dorsal raphe nucleus. J. Comp. Neurol. 474, 364-378.

Erdmann, G., Schutz, G., and Berger, S. (2008). Loss of glucocorticoid receptor function in the pituitary results in early postnatal lethality. Endocrinology 149, 3446-3451.
Chandler, K. J., Chandler, R. L., Southard-Smith, E. M., and

\section{ACKNOWLEDGMENTS}

We thank N.G. Copeland for generously providing EL250 bacterial strains and protocols for PAC targeting in bacteria and S. Gartside for valuable comments on the manuscript. We thank F. Nürnberg for statistical analysis. This work has been supported by the following grants: HEALTH-F2-2007-201714 DEVANX, DFG SFB 636 and DFG STR 699.

\section{SUPPLEMENTARY MATERIAL}

The Supplementary Material for this article can be found online at http://www.frontiersin.org/molecularneuroscience/paper/10.3389/ neuro.02/024.2009/

Feil, R., Wagner, J., Metzger, D., and Chambon, P. (1997). Regulation of Cre recombinase activity by mutated estrogen receptor ligand-binding domains. Biochem. Biophys. Res. Commun. 237, 752-757.

Gaspar, P., Cases, O., and Maroteaux, L. (2003). The developmental role of serotonin: news from mouse molecular genetics. Nat. Rev. Neurosci. 4, 1002-1012.

Geyer, M. A., and Vollenweider, F. X (2008). Serotonin research: contributions to understanding psychoses. Trends Pharmacol. Sci. 29, 445-453.

Giraldo, P., and Montoliu, L. (2001). Size matters: use of YACs, BACs and PACs in transgenic animals. Transgenic Res. 10, 83-103.

Gong, S., Zheng, C., Doughty, M. L., Losos, K., Didkovsky, N., Schambra, U. B., Nowak, N.J., Joyner, A., Leblanc, G., Hatten, M. E., and Heintz, N. (2003). A gene expression atlas of the central nervous system based on bacterial artificial chromosomes. Nature 425, 917-925.

Gordon, J. A., and Hen, R. (2004). Genetic approaches to the study of anxiety. Annu. Rev. Neurosci. 27, 193-222.

Gross, C., and Hen, R. (2004). The developmental origins of anxiety. Nat. Rev. Neurosci. 5, 545-552.

Haller, J., Mikics, E., Halasz, J., and Toth, M. (2005). Mechanisms differentiating normal from abnormal aggression: glucocorticoids and serotonin. Eur. J. Pharmacol. 526, 89-100.

Harfstrand,A., Fuxe, K., Cintra, A., Agnati, L. F., Zini, I., Wikstrom, A. C., Okret, S., Yu,Z.Y., Goldstein, M., and Steinbusch, H. (1986). Glucocorticoid receptor immunoreactivity in monoaminergic neurons of rat brain. Proc. Natl. Acad. Sci. U.S.A. 83, 9779-9783.

Hendricks, T. J., Fyodorov, D. V., Wegman, L. J., Lelutiu, N. B., Pehek, E. A., Yamamoto, B., Silver, J., Weeber, E. J., Sweatt, J. D., and Deneris, E. S. (2003). Pet-1 ETS gene plays a critical role in 5-HT neuron development and is required for normal anxiety-like and aggressive behavior. Neuron 37, 233-247.

Hensler, J. G. (2006). Serotonin. In Basic Neurochemistry, G. J. Siegel RWA, S. T. Brady, D. L. Price, ed. (Burlington, MA, Elsevier Academic Press), pp. 227-248.

Indra, A. K., Warot, X., Brocard, J., Bornert, J.M., Xiao, J.H., Chambon, P., and Metzger, D. (1999). Temporallycontrolled site-specific mutagenesis in the basal layer of the epidermis: comparison of the recombinase activity of the tamoxifen-inducible Cre-ER(T) and Cre-ER(T2) recombinases. Nucleic Acids Res. 27, 4324-4327.

Lanfumey, L., Mongeau, R., Cohen-Salmon, C., and Hamon, M. (2008). Corticosteroid-serotonin interactions in the neurobiological mechanisms of stress-related disorders. Neurosci. Biobehav. Rev. 32, 1174-1184.

Lee, E. C., Yu, D., Martinez de Velasco, J., Tessarollo, L., Swing, D. A., Court, D. L., Jenkins, N. A., and Copeland, N. G. (2001). A highly efficient Escherichia coli-based chromosome engineering system adapted for recombinogenic targeting and subcloning of BAC DNA. Genomics 73, 56-65.

Livet, J., Weissman, T. A., Kang, H., Draft, R. W., Lu, J., Bennis, R. A., Sanes, J. R., and Lichtman, J.W. (2007). Transgenic strategies for combinatorial expression of fluorescent proteins in the nervous system. Nature 450, 56-62.

Lucki, I. (1998). The spectrum of behaviors influenced by serotonin. Biol. Psychiatry 44, 151-162.

Mann, J. J. (2003). Neurobiology of suicidal behaviour. Nat. Rev. Neurosci. 4, 819-828.

Miczek, K. A., de Almeida, R. M., Kravitz, E. A., Rissman, E. F., de Boer, S. F., and Raine,A. (2007). Neurobiology of escalated aggression and violence. J. Neurosci. 27, 11803-11806.

Mossner, R., Muller-Vahl,K.R.,Doring, N., and Stuhrmann, M. (2007). Role of the novel tryptophan hydroxylase-2 gene 
in Tourette syndrome. Mol. Psychiatry 12, 617-619.

Oades, R. D. (2007). Role of the serotonin system in ADHD: treatment implications. Expert Rev. Neurother. 7, 1357-1374.

Pardo, C. A., and Eberhart, C. G. (2007). The neurobiology of autism. Brain Pathol. 17, 434-447.

Schedl, A., Montoliu, L., Kelsey, G., and Schutz, G. (1993). A yeast artificial chromosome covering the tyrosinase gene confers copy number-dependent expression in transgenic mice. Nature 362, 258-261.

Scott, M. M., Wylie, C. J., Lerch, J. K., Murphy, R., Lobur, K., Herlitze, S., Jiang, W., Conlon, R. A., Strowbridge, B. W., and Deneris, E. S. (2005). A genetic approach to access serotonin neurons for in vivo and in vitro studies. Proc. Natl. Acad. Sci. U.S.A. 102, 16472-16477.

Serretti, A., and Mandelli, L. (2008). The genetics of bipolar disorder: genome 'hot regions,' genes, new potential candidates and future directions. Mol. Psychiatry 13, 742-771.

Sodhi, M. S., and Sanders-Bush, E. (2004). Serotonin and brain development. Int. Rev. Neurobiol. 59, 111-174.

Soriano, P. (1999). Generalized lacZ expression with the ROSA26 Cre reporter strain. Nat. Genet. 21, 70-71.

Tronche, F., Kellendonk, C., Kretz, O., Gass, P., Anlag, K., Orban, P. C., Bock, R., Klein, R., and Schutz, G. (1999). Disruption of the glucocorticoid receptor gene in the nervous system results in reduced anxiety. Nat. Genet. 23, 99-103.

Vogt, M. A., Chourbaji, S., Brandwein, C., Dormann, C., Sprengel, R., and Gass, P. (2008). Suitability of tamoxifeninduced mutagenesis for behavioral phenotyping. Exp. Neurol. 211, 25-33.

Wallace, H., Ansell, R., Clark, J., and McWhir, J. (2000). Pre-selection of integration sites imparts repeatable transgene expression. Nucleic Acids Res. 28, 1455-1464.

Walther, D. J., Peter, J.U., Bashammakh, S., Hortnagl, H., Voits, M., Fink, H., and Bader, M. (2003). Synthesis of serotonin by a second tryptophan hydroxylase isoform. Science 299, 76.

Weber, P., Metzger, D., and Chambon, P. (2001). Temporally controlled targeted somatic mutagenesis in the mouse brain. Eur. J. Neurosci. 14, 1777-1783.

Zhuang, X., Masson, J., Gingrich, J. A., Rayport,S., and Hen,R. (2005). Targeted gene expression in dopamine and serotonin neurons of the mouse brain. J. Neurosci. Methods 143, 27-32.

Zill, P., Baghai, T. C., Zwanzger, P., Schule, C., Eser, D., Rupprecht, R., Moller, H. J., Bondy, B., and Ackenheil, M. (2004). SNP and haplotype analysis of a novel tryptophan hydroxylase isoform (TPH2) gene provide evidence for association with major depression. Mol. Psychiatry 9, 1030-1036.
Conflict of Interest Statement: The authors declare that the research was conducted in the absence of any commercial or financial relationships that could be construed as a potential conflict of interest.

Received: 01 September 2009; paper pending published: 24 September 2009; accepted: 14 October 2009; published online: 06 November 2009.

Citation: Weber T, Böhm G, Hermann E, Schütz G, Schönig Kand Bartsch D (2009) Inducible gene manipulations in serotonergic neurons. Front. Mol. Neurosci. 2:24. doi: 10.3389/neuro.02.024.2009

Copyright (c) 2009 Weber, Böhm, Hermann, Schütz, Schönig and Bartsch. This is an open-access article subject to an exclusive license agreement between the authors and the Frontiers Research Foundation, which permits unrestricted use, distribution, and reproduction in any medium, provided the original authors and source are credited. 